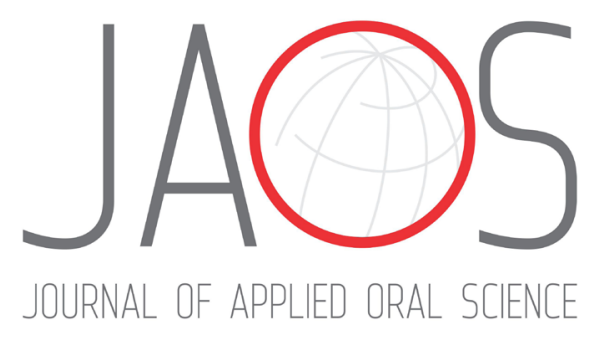

Luana Dutra de CARVALHO ${ }^{1}$

Bernardo Urbanetto PERES ${ }^{2}$

Hazuki MAEZONO 3

Ya SHEN ${ }^{1}$

Markus HAAPASALO ${ }^{1}$

John JACKSON ${ }^{4}$

Ricardo M CARVALHO ${ }^{1}$

Adriana $\mathrm{P} \mathrm{MANSO}^{2}$

\title{
Doxycycline release and antibacterial activity from PMMA/PEO electrospun fiber mats
}

\section{Abstract}

Objective: To investigate the use of polymethyl methacrylate (PMMA) electrospun fiber mats containing different amounts of polyethylene oxide (PEO) as a doxycycline delivery system and to test antibacterial activity against an oral pathogen. Methodology: PMMA powders or PEO (mol wt 200 $\mathrm{Kd})(10,20,30 \% \mathrm{w} / \mathrm{w} /)$ were dissolved in $\mathrm{N}, \mathrm{N}$-dimethylformamide (DMF) to obtain a final polymer concentration of $15 \%$ in DMF (w/v). $2 \%$ Doxycycline monohydrate was added to the solutions and submitted to vortex mixing. The solution was transferred to a plastic syringe and fit into a nanofiber electrospinning unit. The parameters applied were: voltage at $17.2 \mathrm{kV}$; distance of $20 \mathrm{~cm}$ between the needle tip and the collector plate; target speed at $2 \mathrm{~m} / \mathrm{min}$; and transverse speed at $1 \mathrm{~cm} / \mathrm{min}$. Syringe pump speed was 0.15 $\mathrm{mm} / \mathrm{min}$. The drug release analysis was performed by removing aliquots of the drug-containing solution (in PBS) at specific periods. Doxycycline release was quantified using RP-HPLC. Fiber mats from all groups had their antibacterial action tested against $S$. mutans based on inhibition halos formed around the specimens. The experiments were performed in triplicate. Gravimetric analysis at specific periods was performed to determine any polymer loss. Morphological characterization of the electrospun fibers was completed under an optical microscope followed by SEM analysis. Results: The addition of PEO to the PMMA fibers did not affect the appearance and diameter of fibers. However, increasing the \%PEO caused higher doxycycline release in the first $24 \mathrm{~h}$. Fibers containing 30\% PEO showed statistically significant higher release when compared with the other groups. Doxycycline released from the fibers containing $20 \%$ or $30 \%$ of PEO showed effective against S. mutans. Conclusion: The incorporation of PEO at $20 \%$ and $30 \%$ into PMMA fiber mat resulted in effective drug release systems, with detected antibacterial activity against $S$. mutans.

Keywords: Antibacterial. Doxycycline. Drug-release. Electrospinning.

${ }^{1}$ The University of British Columbia, Faculty of Dentistry, Department of Oral Biological and Medical Sciences, Vancouver, Canada

${ }^{2}$ The University of British Columbia, Faculty of Dentistry, Department of Oral Health Sciences, Vancouver, Canada

${ }^{3}$ Osaka University Graduate School of Dentistry Department of Restorative Dentistry and Endodontology, Osaka, Japan

${ }^{4}$ The University of British Columbia, Faculty of Pharmaceutical Sciences, Vancouver, Canada 


\section{Introduction}

Electrospinning is an electrostatic fiber fabrication technique. It has been subject of studies due to its versatility and potential for applications in diverse fields, including tissue engineering, biosensors, filtration, wound dressings, drug delivery, and enzyme immobilization. ${ }^{1,2}$ The sub-micron range spun fibers produced offer various advantages, such as high surface area to volume ratio, tunable porosity (when spun into a mat) and ability to manipulate nanofiber composition to get the properties desired. ${ }^{1}$

Recently, nanofibers have been used in different Dentistry fields, including composite reinforcement, ${ }^{3,4}$ periodontal regeneration, ${ }^{5}$ and implant surface treatments. ${ }^{6}$ They are also used as scaffolds for dental tissue and bone regeneration. ${ }^{7}$ However, the greatest potential of these fibers in Dentistry is the use of mats as drug delivery systems. The ability to produce drugloaded polymeric fiber meshes or mats may offer an enhanced drug release profile compared with other polymeric drug release implants. As in many other fields, this mechanism can be convenient in Dentistry in multiple contexts. ${ }^{8}$

Among several polymers able to form ultrafine fibers in the electrospinning process, polymethyl methacrylate (PMMA) is a reliable biocompatible option, commonly used in restorative Dentistry. ${ }^{9}$ Electrospinning a blend of PMMA and polyethylene oxide (PEO) may result in biocompatible and toughness-enhanced ultra-thin fiber mats very useful for biomedical applications. ${ }^{10}$ PEO is frequently used as a co-spinning component in electrospinning solutions to offset problems related to continuous fiber formation without structural imperfections ${ }^{11}$ or even as a plasticizer to allow fiber formation when blended with different polymers, such as cellulose acetate. ${ }^{12}$

Doxycycline is an antimicrobial drug proved effective against oral bacteria, especially for periodontal disease. ${ }^{13}$ It presents anti-collagenase activity ${ }^{14}$ as additional advantage, which could be beneficial when treating sites sometimes compromised by undue metalloproteinase (MMP) activity such as at bonded interfaces with dentin. ${ }^{15}$

The described polymers formed into fibers loaded with antibacterial and anti-enzymatic drugs, as doxycycline, are expected to be an important controlled drug delivery tool in Dentistry. One considered that the addition of PEO would not affect the homogeneity or diameter or PMMA fibers formed into mats, but that the inclusion of a water soluble polymer such as PEO might increase drug release rates and antibacterial activity of the mats. Thus, the main objectives of this study were to produce PMMA fiber mats using two distinct PMMA powders; and to investigate whether the addition of PEO (200000 Dalton molecular weight) in different concentrations might enhance the release of an antibacterial and anti-MMP drug, the doxycycline.

\section{Methodology}

\section{Fiber mat production}

Polymer fiber mats were produced with two polymethyl methacrylate powders with different molecular weights (PMMA, Mw 75,000 and Mw 996,000, Sigma-Aldrich, St. Louis, MO, USA), dissolved in $\mathrm{N}, \mathrm{N}$-dimethylformamide (DMF, Fisher Scientific, Waltham, MA, USA). Polyethylene oxide (PEO, Mw 200,000, Sigma-Aldrich, St. Louis, MO, USA) was added to the PMMA-DMF solution according to the concentration determined for each group $(0 \%, 10 \%, 20 \%$, or $30 \%$ of PEO). The final polymer concentration was $15 \%(\mathrm{w} / \mathrm{v})$ (Table 1$)$. Each PMMA-PEO-DMF mixture was stirred for $1 \mathrm{~h}$, at $60^{\circ} \mathrm{C}$ to guarantee the dissolution of the polymers. The solution was maintained on the bench at room temperature. Doxycycline monohydrate (SigmaAldrich, St. Louis, MO, USA) was added to a final concentration of $2 \% \mathrm{w} / \mathrm{w}$ and submitted to vortex mixing for $1 \mathrm{~min}$ at $3000 \mathrm{rpm}$. Subsequentially, the vials were left for 10 minutes on the bench top at

Table 1- Concentrations of polymers, solvent, and drug added to the spinning solutions

\begin{tabular}{|c|c|c|c|c|c|}
\hline \multicolumn{6}{|c|}{ Composition } \\
\hline Groups & PMMA75k & PMMA996k & PEO200k & DMF & DOXY \\
\hline 0\%PEO & $0.875 \mathrm{~g}$ & $0.875 \mathrm{~g}$ & - & $10 \mathrm{~g}$ & $0.03 \mathrm{~g}$ \\
\hline $10 \%$ PEO & $0.79 \mathrm{~g}$ & $0.79 \mathrm{~g}$ & $0.17 \mathrm{~g}$ & $10 \mathrm{~g}$ & $0.03 \mathrm{~g}$ \\
\hline $20 \%$ PEO & $0.70 \mathrm{~g}$ & $0.70 \mathrm{~g}$ & $0.35 \mathrm{~g}$ & $10 \mathrm{~g}$ & $0.03 \mathrm{~g}$ \\
\hline $30 \%$ PEO & $0.615 \mathrm{~g}$ & $0.615 \mathrm{~g}$ & $0.52 \mathrm{~g}$ & $10 \mathrm{~g}$ & $0.03 \mathrm{~g}$ \\
\hline
\end{tabular}


room temperature before electrospinning. The solution was transferred to a $10 \mathrm{~mL}$ plastic syringe and fit into a nanofiber electrospinning unit (NEU - Kato Tech, Japan). Electrospinning manufacture of nanofibers depends on many parameters, including the polymer molecular weight and concentration, flow rate, needle/ collector distance, target speed, and applied voltage. Every polymer-drug combination needs to be spun under different conditions to achieve an optimal fiber production. This is usually optimized visually whereby the operator begins with intermediate values for these factors and sees how the polymer spins. In this study, a $15 \%$ polymer solution was associated with non-sputtering spinning and uniform fiber production, which could be seen to "draw" off the needle bead and a white mat appeared on the collection drum. Dry mat samples were collected and observed under high magnification optical microscopy (Olympus Model CX41RF) for uniformity using various flow rates and instrument settings to obtain an optimal fiber mat. The drug was then included in the polymer solution using optimal spinning settings. The final instrument settings were as follows: applied voltage at $17.2 \mathrm{kV}$; distance of $20 \mathrm{~cm}$ between the needle tip and the collector plate; target speed at $2 \mathrm{~m} / \mathrm{min}$; and transverse speed at 1 $\mathrm{cm} / \mathrm{min}$. Syringe pump speed was $0.15 \mathrm{~mm} / \mathrm{min}$ to maintain a clear and visible Taylor cone. Temperature and humidity in the chamber were monitored. Air humidity was kept between $30 \%$ and $50 \%$ with the temperature held constant between 24 and 27 degrees Celsius. The collector plate was covered with an aluminum foil sheet. The fibers were produced over a period of 8 hours and collected randomly, without being aligned. The fiber mats formed were gently removed from the collector plate and stored in sealed plastic bags away from heat and humidity until their use.

\section{Doxycycline release profile}

To analyze the doxycycline release rate from the fiber mats, $10 \mathrm{mg}$ samples of PMMA-PEO-DOXY mats were immersed in vials with $2 \mathrm{~mL}$ of phosphatebuffered saline $\mathrm{pH} 7.4$ (10 mM PBS), which were capped and placed in an incubator at $37^{\circ} \mathrm{C}$ with slow orbital shaking in the dark. The drug release was analyzed by removing $0.7 \mathrm{~mL}$ aliquots of drugcontaining solution of each vial, in specific periods (1 h, 4 h, 8 h, 24 h, 48 h, 168 h). After each sampling, remaining media was replaced with $2 \mathrm{~mL}$ of fresh
PBS. Doxycycline release was quantified using reverse phase High-Performance Liquid Chromatography (with a Waters chromatography system with Millennium software control). The chromatography system used a Novapak $\mathrm{C}^{18}$ column, a $20-\mu \mathrm{L}$ injection volume, detection at $260 \mathrm{~nm}$, and a mobile phase composed of $90 \% \mathrm{pH} 3.4$ potassium phosphate buffer and $10 \%$ acetonitrile, at a flow rate of $1 \mathrm{~mL} / \mathrm{min}$. Calibration graphs were linear in the $6-100 \mu \mathrm{g} / \mathrm{mL}$ concentration range.

\section{Gravimetric analysis}

To measure the relative amount of PEO polymer lost from the mats in aqueous conditions, triplicate samples of each mat were cut in a square format $(1 \times 1 \mathrm{~cm})$ and weighed on an analytical balance (Sartorius ME235S Genius). Thereafter, each sample was submerged in $10 \mathrm{~mL}$ of distilled water, maintained in an incubator at $37^{\circ} \mathrm{C}$ for one of the following periods: $1 \mathrm{~h}, 4 \mathrm{~h}, 8 \mathrm{~h}$, $24 \mathrm{~h}, 48 \mathrm{~h}$, and $168 \mathrm{~h}$. After each period, the samples were removed from water and dried in an incubator at $60^{\circ} \mathrm{C}$ for $24 \mathrm{~h}$. At that point, the remaining samples had their media discarded and $10 \mathrm{~mL}$ of distilled water was added to each vial. The dried samples were weighed, and the numbers were compared with the initial weight. Polymer loss was estimated based on the percentage of the film weight loss for each group.

\section{Antibacterial assay}

Streptococcus mutans (NTCC\# 10449) was used in this study. S. mutans bacterial suspensions was prepared with removal of $20 \mathrm{~mL}$ of $S$. mutans from the stock (frozen at $-80^{\circ} \mathrm{C}$ ). Bacteria were cultured overnight in $5 \mathrm{~mL}$ of brain heart infusion broth (BD TM Bacto ${ }^{\mathrm{TM}}$ BHI, Fischer Scientific, Waltham, MA, USA), at $37^{\circ} \mathrm{C}$ under aerobic conditions. After that, 20-50 $\mu \mathrm{L}$ of the overnight culture was put into $8 \mathrm{~mL}$ of $\mathrm{BHI}$ broth to obtain a second overnight growth solution. For the antibacterial test, $500 \mu \mathrm{L}$ of bacterial solution (diluted $100 \times$ ) was plated on the new BHI agar plate and let dry at room temperature for $1 \mathrm{~h}$. Fiber mat discs with $6 \mathrm{~mm}$ diameter were positioned over the smeared bacteria and incubated overnight at $37^{\circ} \mathrm{C}$. Filter paper (6 $\mathrm{mm}$ diameter) soaked in water and PMMA mats with no drugs were used as negative controls. Filter paper with doxycycline solution (concentration $300 \mu \mathrm{g} /$ $\mathrm{mL}$ ) was used as positive control by soaking the filter paper into the solution and directly applying it to the agar plate with the bacteria. Fiber mats with different concentrations of PEO and the same amount of 
doxycycline had their antibacterial action tested based on inhibition halos formed around the specimens. After the incubation period, the inhibition zones were measured. The experiments were performed in triplicate.

\section{Morphological characterization}

Fiber characteristics were observed under an optical microscope (Olympus Model CX41RF). Scanning electron microscopy (SEM S-2380N, Hitachi, Tokyo, Japan) was used to evaluate the final electrospun mats at an acceleration voltage of $10 \mathrm{kV}$ (SEM S-2380N, Hitachi, Tokyo, Japan). The control mats and the mats used in the release experiment were analyzed to evaluate alterations in fiber structures during drug/ polymer release process. Randomly selected areas of each mat were cut in $5 \times 5 \mathrm{~mm}$ squares and mounted on a stub with carbon tape $(n=3)$. The stubs were then coated with platinum/palladium (Pt/Pd) with an ion sputter coater (Hitachi E-1030 Ion Sputter Coater, Hitachi, Tokyo, Japan). Random images were taken from the selected pieces of each mat at a magnification of $5000 \times$. Average fiber diameter was estimated based on 15 random measurements from pictures taken at the same magnification with image software (Image J, NIH, USA).

\section{Statistical analysis}

Statistical analysis of the data for drug release, weight loss, drug remaining, inhibition halo diameters and fiber diameter were investigated by one-way ANOVA (for each experiment). Level of significance of $\alpha=5 \%$ was set for all statistical analysis. Sigma Plot software (Systat Software, San Jose, USA) was also used.

\section{Results}

\section{HPLC analysis}

Insignificant drug release from PMMA (No PEO content) films was observed. The release of doxycycline from the different films was characterized by a burst phase over the first $24 \mathrm{~h}$, followed by a slow release over the rest of the release study (Figure 1 ). The inclusion of PEO allowed concentration-dependent increase in doxycycline release from PMMA films. By 24 hours, the films had released approximately $1 \mathrm{ug}$, $8 \mathrm{ug}$, or $26 \mathrm{ug}$ of loaded drug (for $10 \%, 20 \%$, and $30 \%$ PEO content films, respectively). After 48 hours, the amount of doxycycline released was very low for all films, but the $30 \%$ PEO loaded films still released approximately $1 \mathrm{ug}$ of drug, more than the other two films. The differences in doxycycline release between the fibers containing $10 \%$ PEO and $20 \%$ PEO compared

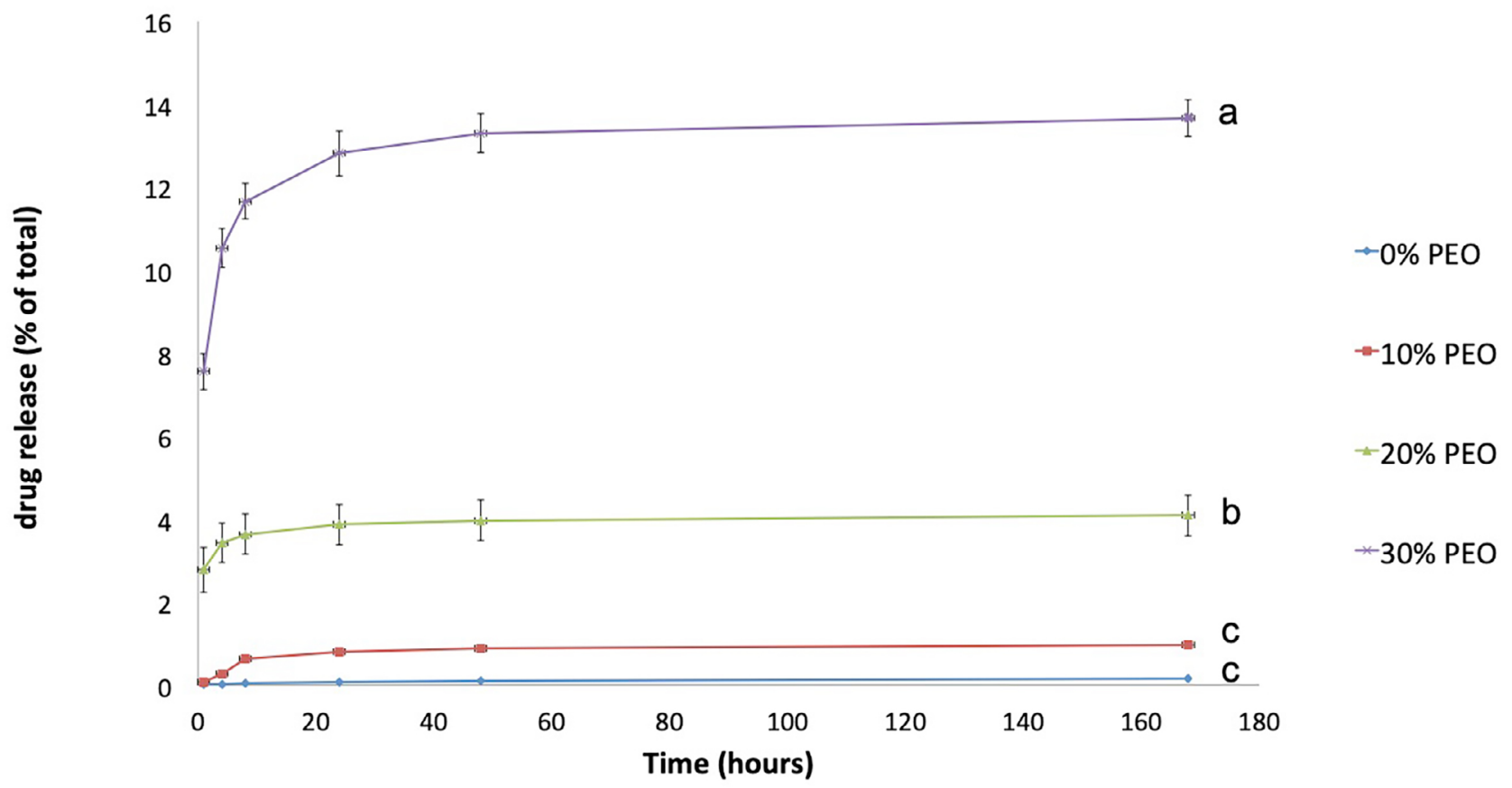

Values with dissimilar letters are significantly different from each other $(p<0.05)$

Figure 1- Release of doxycycline from PMMA fibers $+/$ - PEO at $0 \%, 10 \%, 20 \%$, or $30 \%$ loading 
with $30 \%$ PEO loaded films were statistically significant at all periods up to 48 hours (also, 20\% PEO was higher than $10 \%$ PEO).

\section{Gravimetric analysis}

All fiber mats lost weight over time when placed in water (Figure 2). For 10\% PEO loaded films, weight loss occurred in less than one hour with no further weight loss after that. For $20 \%$ PEO loaded mats, weight loss increased over 8 hours and then plateaued with no further loss. Similarly, for 30\% loading, weight loss was complete at 8 hours. As PMMA is waterinsoluble, the assumption was that all weight loss occurred due to PEO release. For $10 \%$ PEO loaded fiber mats, less than $20 \%$ of encapsulated PEO was released from the fibers. For $20 \%$ and $30 \%$ loadings, almost $50 \%$ of the PEO was released after 8 hours.

\section{Antibacterial analysis}

The initial concentration estimated for $S$. mutans was $5.0 \times 10^{8} \mathrm{CFU} / \mathrm{mL}$. Both $20 \%$ and $30 \%$ PEO loaded doxycycline fibers inhibited the growth of $S$. mutans as seen in Figure 3. These inhibition zones were similar to those resulting from non-encapsulated doxycycline in solution from filter paper.

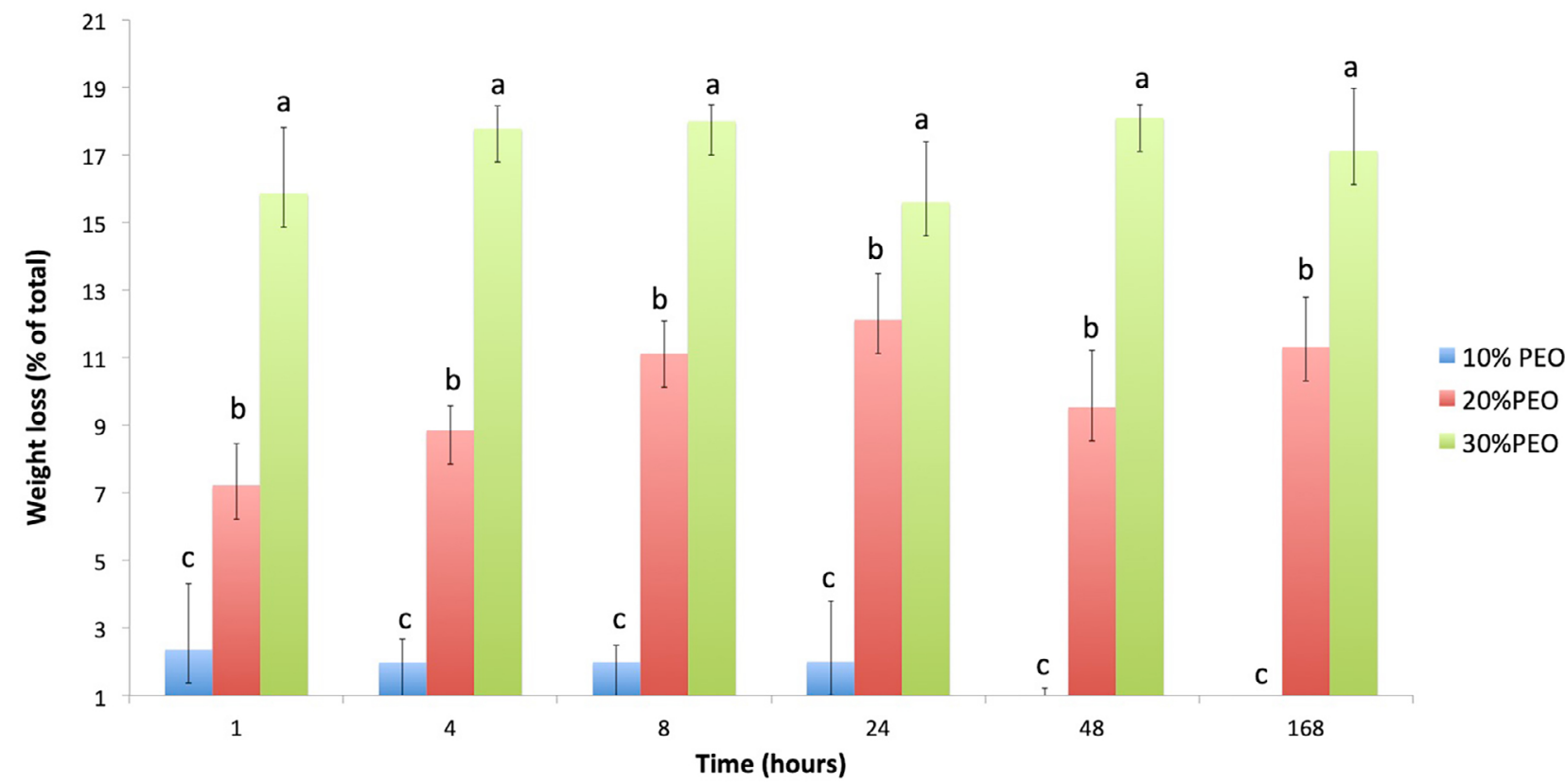

Values with dissimilar letters are significantly different from each other $(p<0.05)$

Figure 2- Percentage of weight lost from the PMMA fibers $+/$ - PEO at $10 \%, 20 \%$, or $30 \%$ loading
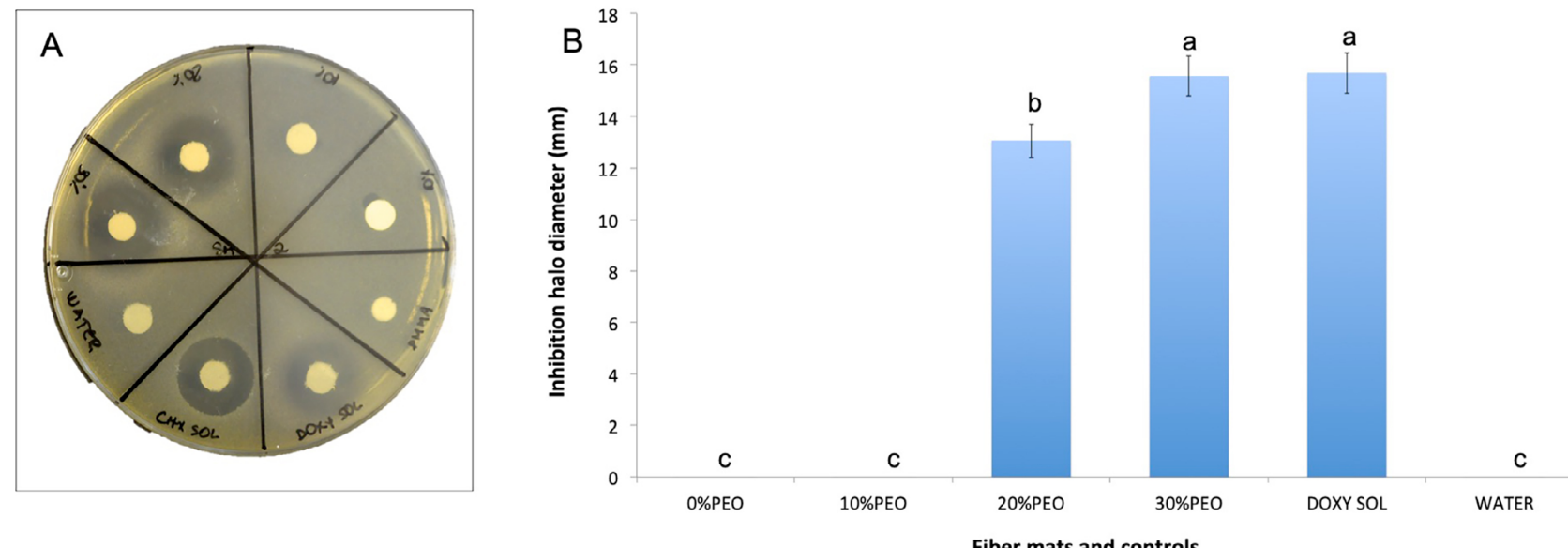

Values with dissimilar letters are significantly different from each other $(p<0.05)$

Figure 3- Comparison of inhibition halo size $(\mathrm{mm})$ against $S$. mutans formed around the different fiber mats and controls. 3A: Image of inhibition halos; 3B: graphic representation of inhibition halo measurements $(\mathrm{mm})$ 


\section{Morphological characterization}

SEM images showed randomly oriented fibers in all groups (Figure 4). All PMMA fiber mats presented homogenous fiber distribution with a uniform diameter and no drug aggregates or imperfections. The mean fiber diameter (standard deviation) of all groups is presented in Figure 5. One-way ANOVA showed the different PMMA/PEO ratio and immersion in PBS for up to 7 days did not affect significantly fiber diameter. (Figure 4 and Figure 5)

\begin{tabular}{|c|c|c|c|}
\hline Control & $10 \%$ PEO & $20 \%$ PEO & $30 \% P E O$ \\
\hline 24h immersed & & \\
\hline
\end{tabular}

Figure 4- Scanning electron micrographs of PMMA control fibers (top left) and PMMA fibers containing $10 \%, 20 \%$, and $30 \%$ PEO and $2 \%$ doxycycline before immersion in PBS, and after 24 hours and 7 days immersed (in sequence from top to bottom)

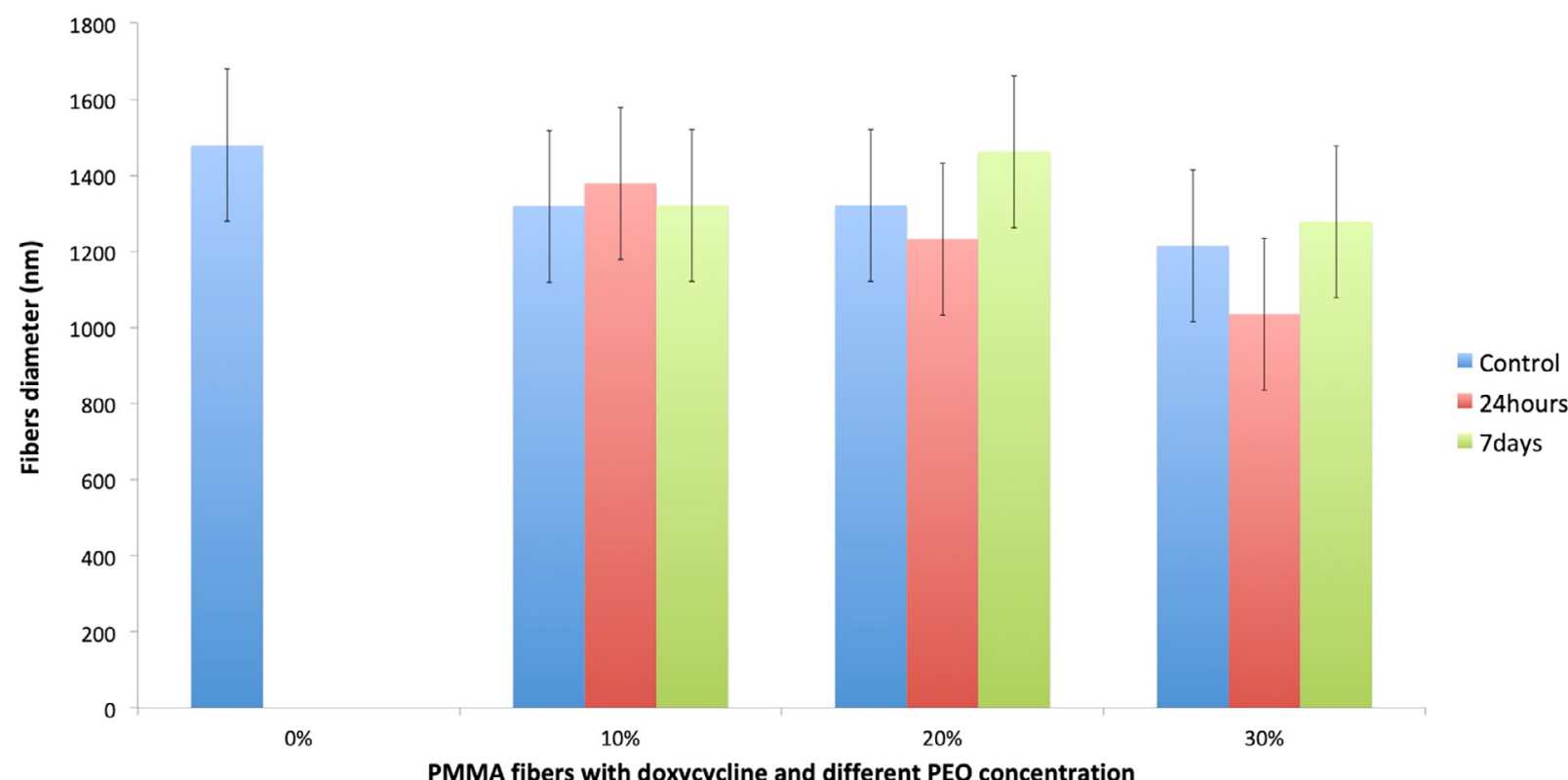

No significant differences were found among the experimental groups $(p>0.05)$

Figure 5- Fiber diameter (nm) with different PEO concentrations at post-spinning, 24 hours, and 7 days water immersion 


\section{Discussion}

This study showed the doxycycline release profile was significantly affected by PEO amount present in PMMA fibers.

The manufacture and characterization of doxycycline loaded PMMA fiber mats by electrospinning are described in this study. Although other methods, such as pressurized gyratory spinning, are described as efficient methods for drug delivery purposes ${ }^{16}$, the method chosen provides an efficient encapsulation for the polymer PMMA, which is extensively used for dental applications. The electrospinning used in our laboratory allowed control of manufacturing variables for fiber optimization and mat production. Therefore, this method was selected for the manufacture of doxycycline fiber mats.

Generally, the incorporation of high molecular weight PEO polymer does not interfere with fiber production, since the long molecular weight molecules may align and help to electrospin the long PMMA molecules. At PMMA to PEO ratios of 70:30, the fiber chains (and mats) were strong and uniform in nature, demonstrating that high PEO incorporation levels might be achieved. ${ }^{17}$ FTIR is frequently used to characterize polymer blends but, in this case, the strong IR band arising from the $\mathrm{C}-\mathrm{O}$ group is present in both PMMA and PEO. Reddy, et al. ${ }^{18}$ (2017) showed that the incorporation of PEO in PMMA films only resulted in a minor broadening of $\mathrm{C}-\mathrm{O}$ stretch band at $1152 \mathrm{~cm}^{-1}$ wavenumber and that other variations in IR spectra were minor. Therefore, FTIR analysis was not performed in this study. In general, water-soluble antibiotics release very slowly from PMMA. PEO is reported as a carrier polymer that provides molecular entanglement required for electrospinning. Jackson, et al. showed the addition of water soluble molecules, such as sodium chloride, dextran or PEO, to PMMA bone cement monolithic blocks only caused minor changes in strength, but greatly enhanced the drug release profiles, such as vancomycin and linezolid. ${ }^{19}$ In another study, the addition of PEO to drug loaded polycaprolactone implants enhanced drug release profiles as the PEO dissolved out of hydrophobic brittle polycaprolactone. ${ }^{20}$ Whilst the insertion of PEO into cement is easy (simple mixing), electrospinning methods might be excessively compromised by the addition of large amounts of excipients. Electrospinning PMMA with PEO for enhanced drug release purposes overcomes a technical hurdle with positive results.

To the best our knowledge, no reports showed the addition of different percentages of PEO might affect doxycycline release profile from electrospun fibers. The hypothesis is that the release of significant PEO amounts from PMMA might open up the matrix slightly, so that water can access deeper fiber areas to solubilize and release doxycycline. Blended fibers using hydrophobic and hydrophilic polymers as PMMA and PEO may produce a prolonged and controlled release system for other drugs. ${ }^{21,22}$ The large surface area, associated with spun fabrics, allows fast and efficient solvent evaporation, providing incorporated drug limited time to recrystallize, which favors the formation of amorphous dispersions or solid solutions of drug in polymer. ${ }^{23}$

PMMA is a well-known polymer, commonly called acrylic, used for temporary crowns, complete or partial dentures, orthodontic appliances, and personalized trays for impressions among its dental applications. Its qualities of biocompatibility, reliability, relative ease of manipulation, and low toxicity were soon seized upon and incorporated by many different medical specialties. ${ }^{24,25}$ PMMA is hydrophobic and dimensionally stable. Therefore, it is a versatile polymer in drug delivery systems and has been used in various areas of the biomedical field: it has been proven to be an efficient antimicrobial agent delivery device, preventing in vivo Staphylococcus aureus biofilm formation. ${ }^{26}$ On the other hand, as this is a hydrophobic polymer, it does not swell at all in water. Other biocompatible polymers, such as poly (lacticco-glycolic) (PLGA), are more commonly used in electrospinning methods for drug delivery because they swell in water and release drugs over a long period, until degradation. ${ }^{27,28}$. However, polymers that distort in water are not suitable for most dental applications requiring mechanical stability.

In this study, we showed little change in fiber diameter after the immersion of mats in water, making even 30\% PEO loaded mats suitable for producing dimensionally stable fibers.

$\mathrm{PEO}$ is a polymer with high solubility in both aqueous and organic solvents. It is suitable for biological applications mostly because of its water solubility and low intrinsic toxicity. ${ }^{29}$ The high hydrophilic nature of PEO may increase the permeation of drugs when conjugated with them. It also enhances the physical and chemical stability of drugs and prevents 
the aggregation of drugs in vivo because of steric hindrance and/or masking of charges provided through the formation of a conformational cloud. ${ }^{30}$ Due to those characteristics, PEO was selected and suggested to act as a drug delivery polymer in this study.

The association of PMMA with other polymers, such as PEO, polyvinyl alcohol, and polycaprolactone $(P C L)$, to achieve an ideal drug release profile has been previously shown. ${ }^{10,31,32}$ This study tested different PMMA/PEO ratios and their impact on doxycycline release profile. It was observed that doxycycline release increased as the percentage of PEO in fibers increased. Doxycycline release increased significantly when $30 \%$ PEO was added. When $10 \%$ PEO was added, the release was not significantly different from that of the control ( $0 \%$ PEO). It suggests doxycycline remained trapped within the fibers and strongly indicates the requirement of a minimal amount of PEO in fiber to allow the release of significant amounts of doxycycline. The relative contribution and the crucial role of PEO dissolution in the release of doxycycline correlates with the lower weight loss observed for the 10\% PEO group.

Morphological characteristics of the fibers, such as diameter, may have a significant impact on the drug release. ${ }^{33}$ In this study, it was initially challenging to produce uniform, beadless fiber mats from PMMA. A pilot study revealed that the best formulation combined two different molecular weights of PMMA (75K and 996K) in pure DMF. The electrospun fibers formed were uniform, well distributed, and beadless. The fiber mats obtained were easy to handle. No statistically significant difference was found in the mean diameter among the groups. Although the fibers were relatively large in diameter (ca.>1000 nm) considering that nano level fibers can be produced, they were uniform among the groups and allowed us to interpret our findings based exclusively on the PEO concentration variable. Reproducibility and uniformity are more important than reduced diameter. ${ }^{9}$

Multiple alternative strategies to the use of antibiotics have been applied to enhance antimicrobial activity of polymeric fiber meshes, including the incorporation of antimicrobial metals, semi-metals, or graphene compounds. ${ }^{34,35}$. In this study, doxycycline was the drug selected for the experiment because it is a broad-spectrum antibiotic drug, which has been shown to be active against both gram-positive and gram-negative organisms. Streptococcus mutans was selected due to its clinical relevance on metabolic-induced dental tissue loss. Doxycycline has been successfully used to control the progression of periodontal disease by systemic and localized delivery at antimicrobial levels. The drug has also been encapsulated within nanotube-modified dentin adhesive, presenting sub-antimicrobial concentrations and ability to inhibit MMPs. ${ }^{13,36-39}$

In future studies using PMMA/PEO fiber, drug release profiles for specific dental applications need to be better quantified. However, the concept tested herein can open multiple doors for future studies aiming at validating the clinical application of doxycycline loaded PMMA-PEO mats as drug delivery systems. An example of a good clinical application for these mats could be their use as pulp protection and antibacterial materials for restorative procedures on deep cavities, protecting the pulp tissue and, simultaneously, acting as antibacterial and anti-MMP agent. Currently, an option for this purpose relies on calcium hydroxide, which may not provide an effective long-term seal against bacterial factors. ${ }^{40}$ This method would be an alternative approach as the material could release not just antibiotics, but also inductors of calcification or anti-inflammatory agents in future productions.

\section{Conclusion}

Based on the experiments performed, one can conclude that consistent production of PMMA/ PEO eletrospun fiber mats with adequate handling properties to be used in dental applications was possible. PEO inclusion influenced positively the doxycycline release profile from PMMA fiber mats, but did not alter the morphological characteristics of the fibers. PMMA fiber mats containing $20 \%$ and $30 \%$ PEO and $2 \%$ doxycycline presented antibacterial activity against $S$. mutans.

\section{References}

1- Bhardwaj N, Kundu SC. Electrospinning: A fascinating fiber fabrication technique. Biotechnol Adv. 2010;28(3):325-47. doi:10.1016/j. biotechadv.2010.01.004

2- Shabafrooz V, Mozafari M, Vashaee D, Tayebi L. Electrospun nanofibers: from filtration membranes to highly specialized tissue engineering scaffolds. J Nanosci Nanotechnol. 2014;14:522-34. 
3- Vidotti HA, Manso AP, Leung V, Valle AL, Ko F, Carvalho RM. Flexural properties of experimental nanofiber reinforced composite are affected by resin composition and nanofiber/resin ratio. Dent Mater Off Publ Acad Dent Mater. 2015;31:1132-41. doi:10.1016/j.dental.2015.06.018 4- Sun W, Cai Q, Li P, Deng X, Wei Y, Xu M, et al. Post-draw PAN-PMMA nanofiber reinforced and toughened Bis-GMA denta restorative composite. Dent Mater. 2010;26:873-80. doi:10.1016/j. dental.2010.03.022

5- Chaturvedi TP, Srivastava R, Srivastava AK, Gupta V, Verma PK. Doxycycline poly e-caprolactone nanofibers in patients with chronic periodontitis: a clinical evaluation. J Clin Diagn Res JCDR. 2013;7:233942. doi: $10.7860 / J C D R / 2013 / 5858.3519$

6- Bottino MC, Münchow EA, Albuquerque MTP, Kamocki K, Shahi R, Gregory RL, et al. Tetracycline-incorporated polymer nanofibers as a potential dental implant surface modifier. J Biomed Mater Res B Appl Biomater. 2017;105:20185-92. doi:10.1002/jbm.b.33743

7- Bottino MC, Yassen GH, Platt JA, Labban N, Windsor LJ, Spolnik KJ, et al. A novel three-dimensional scaffold for regenerative endodontics: materials and biological characterizations. J Tissue Eng Regen Med. 2015;9:E116-23. doi:10.1002/term.1712

8- Ignatious F, Sun L, Lee C-P, Baldoni J. Electrospun nanofibers in oral drug delivery. Pharm Res. 2010;27:576-88. doi:10.1007/s11095010-0061-6

9- Uyar T, Çökeliler D, Doğan M, Koçum IC, Karatay O, Denkbaş EB. Electrospun nanofiber reinforcement of dental composites with electromagnetic alignment approach. Mater Sci Eng C Mater Biol Appl. 2016;62:762-70. doi:10.1016/j.msec.2016.02.001

10- Andersson RL, Martínez-Abad A, Lagaron JM, Gedde UW, Mallon $\mathrm{PE}$, Olsson RT, et al. Antibacterial properties of tough and strong electrospun PMMA/PEO fiber mats filled with lanasol: a naturally occurring brominated substance. Int J Mol Sci. 2014;15:15912-23. doi:10.3390/ijms150915912

11- Kazemi Pilehrood M, Dilamian M, Mirian M, Sadeghi-Aliabadi H, Maleknia L, Nousiainen $P$, et al. Nanofibrous chitosan-polyethylene oxide engineered scaffolds: a comparative study between simulated structural characteristics and cells viability. BioMed Res Int. 2014;2014: 438065. doi: $10.1155 / 2014 / 438065$

12- Chen L, Bromberg L, Hatton TA, Rutledge GC. Electrospun cellulose acetate fibers containing chlorhexidine as a bactericide. Polymer. 2008;49:1266-75. doi:10.1016/j.polymer.2008.01.003

13- Bostanci N, Belibasakis GN. Doxycycline inhibits TREM-1 induction by Porphyromonas gingivalis. FEMS Immunol Med Microbiol. 2012;66:37-44. doi:10.1111/j.1574-695X.2012.00982.x

14- Nordström D, Lindy O, Lauhio A, Sorsa T, Santavirta S, Konttinen YT. Anti-collagenolytic mechanism of action of doxycycline treatment in rheumatoid arthritis. Rheumatol Int. 1998;17:175-80.

15- Freitas PH, Giannini M, Consani S, Deng S, Franca R. Effect of doxycycline incorporated within dental adhesives on tooth-restoration interface. Dent Mater. 2016;32:e87. doi:10.1016/j.dental.2016.08.181 16- Heseltine PL, Ahmed J, Edirisinghe M. Developments in pressurized gyration for the mass production of polymeric fibers. Macromol Mater Eng. 2018;303:1800218. doi:10.1002/mame.201800218

17- Andersson RL, Ström V, Gedde UW, Mallon PE, Hedenqvist MS, Olsson RT. Micromechanics of ultra-toughened electrospun PMMA/PEO fibres as revealed by in-situ tensile testing in an electron microscope. Sci Rep. 2014;4:6335. doi:10.1038/srep06335

18- Reddy MR, Reddy MJ, Subrahmanyam AR. Structural, thermal and optical properties of PMMA, PEO and PMMA/PEO/LiCIO4 polymer electrolyte blends. Mater Sci Res India. 2017;14:123-7.

19- Jackson J, Leung F, Duncan C, Mugabe C, Burt H. The use of bone cement for the localized, controlled release of the antibiotics vancomycin, linezolid, or fusidic acid: effect of additives on drug release rates and mechanical strength. Drug Deliv Transl Res. 2011;1:121-31. doi:10.1007/s13346-011-0015-5
20- Nien Y-H, Shih C-Y, Yang C-Y, Lu C-J, Ye Q-X. Preparation and characterization of electrospun polycaprolactone/polyethylene oxide membranes. J Polym Res. 2013;20:166. doi:10.1007/s10965-0130166-9

21- Zupančič Š, Sinha-Ray S, Sinha-Ray S, Kristl J, Yarin AL. Long-term sustained ciprofloxacin release from PMMA and hydrophilic polymer blended nanofibers. Mol Pharm. 2016;13:295-305. doi:10.1021/acs. molpharmaceut.5b00804

22- Poormasjedi-Meibod MS, Pakyari M, Jackson JK, Salimi Elizei S, Ghahary A. Development of a nanofibrous wound dressing with an antifibrogenic properties in vitro and in vivo model. J Biomed Mater Res A. 2016;104:2334-44. doi:10.1002/jbm.a.35770

23- Verreck G, Chun I, Peeters J, Rosenblatt J, Brewster ME. Preparation and characterization of nanofibers containing amorphous drug dispersions generated by electrostatic spinning. Pharm Res. 20:810-7. doi:10.1023/A: 1023450006281

24- Frazer RQ, Byron RT, Osborne PB, West KP. PMMA: an essential material in medicine and dentistry. J Long Term Eff Med Implants. 2005; 15:629-39.

25- Vargas KF, Borghetti RL, Moure SP, Salum FG, Cherubini K, Figueiredo MAZ. Use of polymethylmethacrylate as permanent filling agent in the jaw, mouth and face regions--implications for dental practice. Gerodontology. 2012;29:e16-22. doi:10.1111/j.17412358.2011.00479.x

26- Anguita-Alonso P, Giacometti A, Cirioni O, Ghiselli R, Orlando F, Saba $\mathrm{V}$, et al. RNAIII-Inhibiting-Peptide-Loaded polymethylmethacrylate prevents in vivo Staphylococcus aureus biofilm formation. Antimicrob Agents Chemother. 2007;51:2594-6. doi:10.1128/AAC.00580-06 27- Chen DW, Hsu Y-H, Liao J-Y, Liu S-J, Chen J-K, Ueng SW-N. Sustainable release of vancomycin, gentamicin and lidocaine from novel electrospun sandwich-structured PLGA/collagen nanofibrous membranes. Int J Pharm. 2012;430:335-41. doi:10.1016/j. ijpharm.2012.04.010

28- Tseng Y-Y, Kao Y-C, Liao J-Y, Chen W-A, Liu S-J. Biodegradable drug-eluting poly[lactic-co-glycol acid] nanofibers for the sustainable delivery of vancomycin to brain tissue: in vitro and in vivo studies. ACS Chem Neurosci. 2013;4:1314-21. doi:10.1021/cn400108q

29- Thakur VK, Thakur MK. Handbook of sustainable polymers: processing and applications. CRC Press; 2016.

30- Knop K, Hoogenboom R, Fischer D, Schubert US. Poly(ethylene glycol) in drug delivery: pros and cons as well as potential alternatives. Angew Chem Int Ed Engl. 2010;49:6288-308. doi:10.1002/ anie. 200902672

31-Zupančič Š, Sinha-Ray S, Sinha-Ray S, Kristl J, Yarin AL. Controlled release of ciprofloxacin from core-shell nanofibers with monolithic or blended core. Mol Pharm. 2016;13:1393-404. doi:10.1021/acs. molpharmaceut.6b00039

32- Srikar R, Yarin AL, Megaridis CM, Bazilevsky AV, Kelley E. Desorption-limited mechanism of release from polymer nanofibers. Langmuir ACS J Surf Colloids. 2008;24:965-74. doi:10.1021/la702449k 33- Chen SC, Huang XB, Cai XM, Lu J, Yuan J, Shen J. The influence of fiber diameter of electrospun poly(lactic acid) on drug delivery. Fibers Polym. 2012;13:1120-5. doi:10.1007/s12221-012-1120-x 34- Matharu RK, Charani Z, Ciric L, Illangakoon UE, Edirisinghe M. Antimicrobial activity of tellurium-loaded polymeric fiber meshes. J Appl Polym Sci. 2018;135:46368. doi:10.1002/app.46368 35- Matharu RK, Porwal H, Ciric L, Edirisinghe M. The effect of graphene-poly(methyl methacrylate) fibres on microbial growth. Interface Focus. 2018;8:20170058. doi:10.1098/rsfs.2017.0058 36- Feitosa SA, Palasuk J, Kamocki K, Geraldeli S, Gregory RL, Platt JA, et al. Doxycycline-Encapsulated Nanotube-Modified Dentin Adhesives. J Dent Res. 2014;93(12):1270-1276. doi:10.1177/0022034514549997 
37- Botelho MA, Martins JG, Ruela RS, Queiroz DB, Ruela WS. Nanotechnology in ligature-induced periodontitis: protective effect of a doxycycline gel with nanoparticules. J Appl Oral Sci Rev FOB. 2010;18(4):335-342.

38- Choi D-H, Moon I-S, Choi B-K, Paik JW, Kim YS, Choi SH, et al. Effects of sub-antimicrobial dose doxycycline therapy on crevicular fluid MMP-8, and gingival tissue MMP-9, TIMP-1 and IL-6 levels in chronic periodontitis. J Periodontal Res. 2004;39(1):20-26.
39- Caton J, Ryan ME. Clinical studies on the management of periodontal diseases utilizing subantimicrobial dose doxycycline (SDD). Pharmacol Res. 2011;63:114-20. doi:10.1016/j.phrs.2010.12.003

40- Schuurs AH, Gruythuysen RJ, Wesselink PR. Pulp capping with adhesive resin-based composite vs. calcium hydroxide: a review. Endod Dent Traumatol. 2000;16:240-50. 